\title{
Mapant Spain: an automatically generated orienteering map for Spain
}

\author{
Manuel Jurado Alonso a , Agustín Caballero Belda a , Javier Arufe Varela a , Jesús María Garrido \\ Sáenz de Tejada ${ }^{\mathrm{b}}$, Jesús Moreno Jabato ${ }^{\mathrm{b}}$ \\ a Spanish Orienteering Federation(FEDO),atoja92@gmail.com,agus.caballero.belda@gmail.com,jarufe.geo@yahoo.com \\ ${ }^{b}$ Instituto Geográfico Nacional (IGN),jmgarrido@mitma.es,jmjabato@mitma.es
}

Keywords: Orienteering, outdoor, cartography, LiDAR, collaborative.

\begin{abstract}
:
Orienteering is a sport in which the competitor (orienteer) completes a course in the shortest possible time, by navigating to control points aided only by a map and compass. An orienteering map is a topographical map. It assists navigation through the terrain by representing a selection of prominent features. These features are of great importance to show the shape of the ground, and this in turn assists the orienteer with serve route choice by showing variations in runnability (which affects speed) and visibility. For this purpose, orienteering maps provide us valuable information about contour lines, cliffs or vegetation density to name a few. Due to these characteristics, we can discover new places and experience endless adventures through the communication this kind of cartography brings us.
\end{abstract}

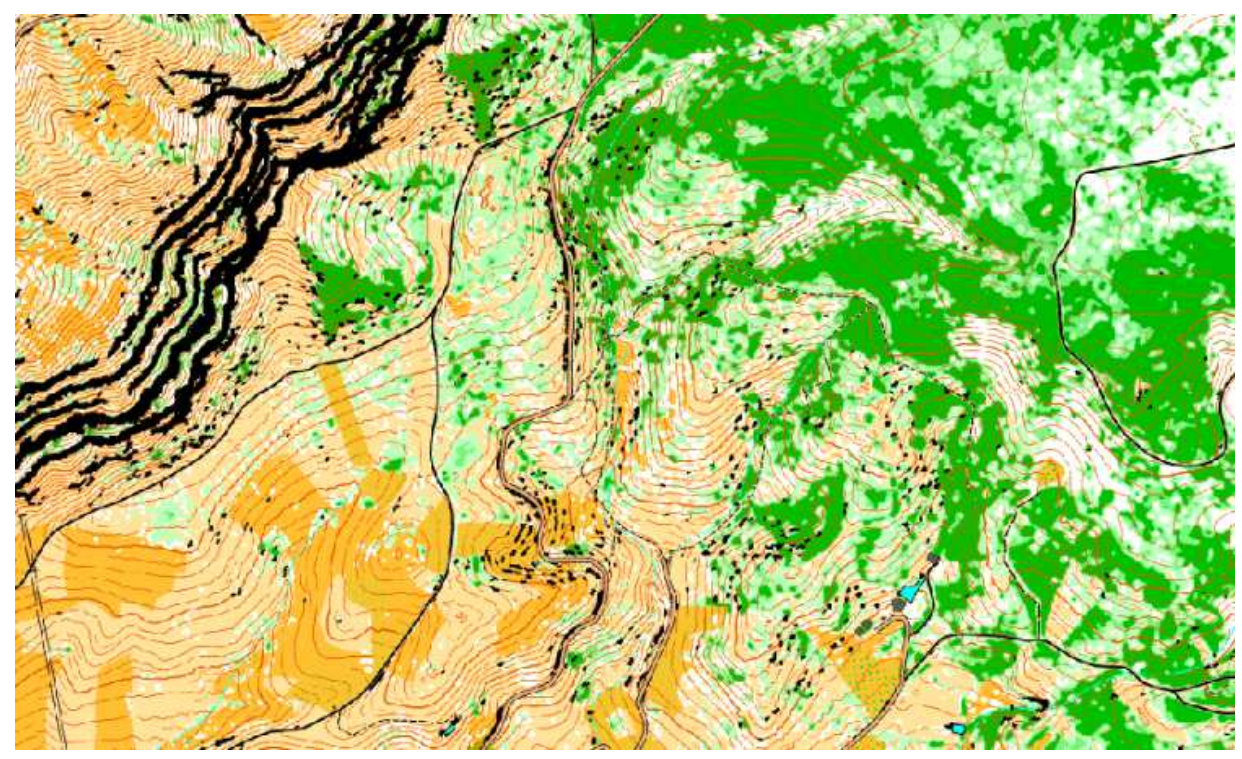

Figure 1. Mapant mountain area.

Mapant Spain was born from the idea of creating an orienteering map of Spain $\left(505.000 \mathrm{~km}^{2}\right)$ generated automatically. The first Mapant produced in Finland back in 2016 has inspired this project (www.mapant.fi). The ambition of covering the whole territory of Spain in a single map would not have been possible without public access to geographic data. The project is a collaboration between the Spanish Orienteering Federation (FEDO) and the Spanish National Geographic Institute (IGN). A part from these two organizations, a group of volunteers (all of them from the orienteering field) has participated offering their computes for the processing. Discovering our territory, promoting outdoor activities (including orienteering, rogaine and hiking) as well as providing information that is more accurate to geographers or rescue and emergency staff are some of the main uses of Mapant.

The main sources for the generation of the map have been both LiDAR (LAZ) and geographic vector data. The LiDAR used comes from the national LiDAR program of Spain (PNOA-LiDAR), data from both 1st (2009-2015) and 2nd (2015-2020) coverage (point cloud density varies from 0.5 to 4 points/m2) have been used. Vector data comes mainly from the National Topographic Map (BTN25) and also from the Agricultural Geographic Information System (SIGPAC) and Open Street Map (OSM). 
A collaborative workflow method was used for data processing. The whole of Spain has been divided into $1 \mathrm{x} 1 \mathrm{~km}$ tiles and then each user/volunteer processed as many tiles as his/her PC could handle. For an average PC it would have taken approximately 14 years to process the whole territory of Spain. For the point cloud data pre-processing, LAStools from Martin Isenburg (las2las and las2txt tools, under LGPL 2.1 license) has been used. For the generation of contours, cliffs and vegetation, Karttapullautin from Jarkko Ryppo has been used (free software for non-commercial or for navigation sport purposes). The symbology used corresponds to Foot Orienteering ISOM- 2017-2 standards. Due to the map being $100 \%$ automatically generated, few symbols have been modified to improve legibility.

In order to do collaborative processing, a software call MapAnt Client was created. This software allows the user to download and process LiDAR automatically, to render the map with the symbology that corresponds for an orienteering map and to upload the generated map to the server. As was mentioned previously, the users are a group of volunteers offering their computers for processing simultaneously. This collaborative way of working allowed the project to reach a peak of 50 volunteers/PC processing simultaneously up to 2.500 tiles per hour.

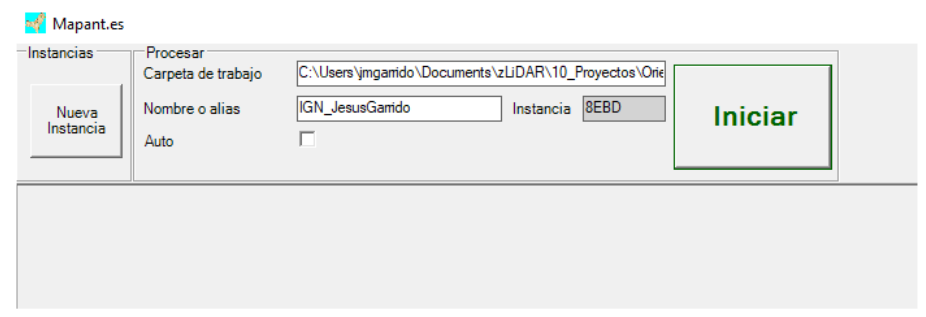

Figure 2. MapAnt Client.

Once the tile has been rendered through this process, MapAnt Client generates a PNG file (in this case in UTM 28/29/30/31 depending of the area) for each tile. When an area of approximately $30 \times 20 \mathrm{~km}$ (this area corresponds to a 1:50.000 section of the National Topographic Map) is completed, the server merges (using GDAL tools) all of those corresponding PNG files in a single GeoTIFF (COG, EPSG:3857), which are the files included in the viewer. Once the project is completed, there will be about 1.000 GeoTIFF files. The user can download a section of the map in PNG format (georeferenced) from the viewer. This downloaded image is formed from the original images in MapAnt Client which allows the final image to maintain a higher resolution (600ppi).

Due to the complexity of the generation of the vector data (for example, a complex algorithm is used to avoid duplicity of trails that appear in different sources), downloading a final image must be done through the MapAnt Client. The file generated can be opened with the software Open Orienteering Mapper (OOM). OOM is a collection of tools which help with the creation of orienteering maps and the organization of orienteering events.

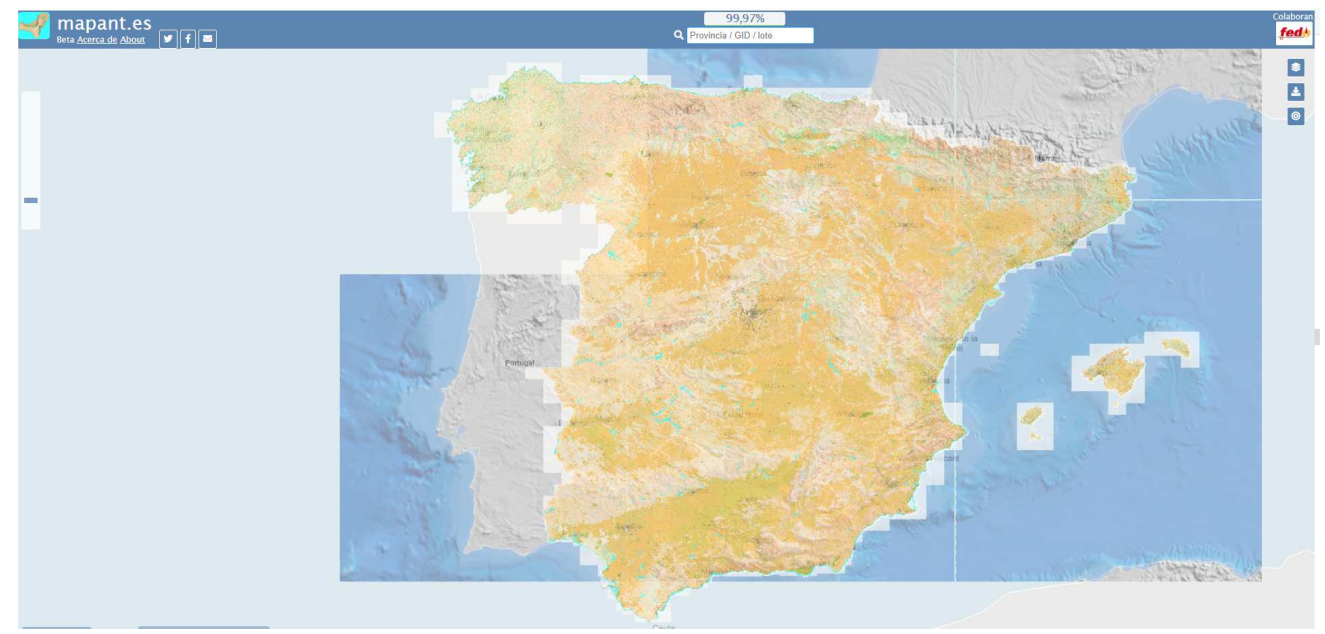

Figure 3. mapant.es viewer.

At the moment of writing this abstract, version 1.0 of Mapant is being finalized. The development and processing of the project has taken about 6 months. The processing time adding up all the computers have been about 80.0000 hours $(9$ years and 3 months), without taking into account the reprocessing time of some areas. The product will be offered in the viewer, as WMS and in image format through the Download Center of the National Centre for Geographic Information (CNIG). 